\title{
Comparison of Tooth Discoloration Induced by Calcium- enriched Mixture, Mineral Trioxide Aggregate, and Endocem
}

\author{
Robab Farhang ${ }^{1}$, Somayeh Hekmatfar ${ }^{2}$, Vahid Samadi ${ }^{3}$, Avisa Meraji ${ }^{4}$, Karim Jafari ${ }^{5}$
}

\begin{abstract}
Aim: Dental discoloration after the application of endodontic materials has become a primary cause of concern for many dental patients in recent times. Regarding this, the present in vitro study was performed to evaluate tooth discoloration induced by calcium-enriched mixture (CEM) cement, mineral trioxide aggregate (MTA), and Endocem.

Materials and methods: This study was conducted on 54 healthy central incisors extracted due to orthodontic treatment or periodontal disease. The samples were assigned into three groups in which the pulp chambers were filled with Endocem (group I), CEM cement (group II), and MTA (group III). The samples were sealed by glass ionomer cement. Color change was measured using the Vita Easyshade spectrophotometer at the baseline as well as 30 and 90 days after the application of cements. Data analysis was performed using SPSS software (version 23).

Results: A visible color change with a delta of $>3.3$ was observed in all groups 1 month postintervention. The results revealed a significant difference among the three groups in terms of color change 1 month after the intervention ( $p$ value $<0.001$ ). In this regard, group I and group II showed the highest and lowest color change, respectively. However, there was no significant difference 3 months after the application of the cements.

Conclusion: As the findings indicated, tooth discoloration was similarly detectable 1 month after the application of CEM cement, MTA, and Endocem.

Clinical significance: CEM cement, MTA, and Endocem showed similar tooth discoloration after 3 months placement.

Keywords: Calcium-enriched mixture, Crown discoloration, Endocem, Mineral trioxide aggregate, Spectrophotometer.

World Journal of Dentistry (2020): 10.5005/jp-journals-10015-1758
\end{abstract}

\section{INTRODUCTION}

Tooth discoloration has become a primary cause of concern for many dental patients in recent times. Discoloration is any change in the color of a tooth, either externally or internally, which presents a major Esthetic problem, especially if it involves the anterior teeth. ${ }^{1}$ Mineral trioxide aggregate (MTA) is a bioactive and biocompatible endodontic cement substance enjoying low cytotoxicity and solubility, as well as high sealing ability, compared to the conventional endodontic materials. ${ }^{2,3}$

The MTA is derived from Portland cement, composed of fine hydrophilic particles of tricalcium silicate, tricalcium aluminate, tricalcium oxide, and other mineral oxides. This material is set when located in a moist context. This moisture leads to the generation of a crystallized calcium silicate hydrate gel as well as calcium hydroxide. ${ }^{4}$ According to the evidence, MTA can be efficiently applied in direct and indirect pulp capping, vital pulp therapy, and partial and complete pulpotomy. 5 ,6

Gray MTA (gMTA) was primarily developed; however, it induces tooth discoloration. ${ }^{7,8}$ Therefore, in order to eliminate this issue, white MTA (WMTA) was developed by removing or lowering the concentration of various metal oxides accounting for color change. ${ }^{9}$ However, white MTA also resulted in tooth discoloration the same as gray formulations. ${ }^{5,10}$

In recent years, pozzolan cement-based MTA (Endocem MTA; Maruchi, Wonju, Korea) has been proposed as a new MTA type for endodontic purposes. The merits of Endocem MTA include improved manipulation properties, decreased setting time, and identical biocompatibility and osteogenicity/odontogenicity. ${ }^{11,12}$

Calcium-enriched mixture (CEM) is another biomaterial with clinical applications similar to those of MTA. ${ }^{13,14}$ This material enjoys
${ }^{1}$ Department of Endodontics, Faculty of Dentistry, Ardabil University of Medical Sciences, Ardabil, Iran

${ }^{2}$ Department of Pediatric Dentistry, Faculty of Dentistry, Ardabil University of Medical Sciences, Ardabil, Iran

${ }^{3,4}$ Faculty of Dentistry, Ardabil University of Medical Sciences, Ardabil, Iran

${ }^{5}$ Department of Prosthodontics, Faculty of Dentistry, Ardabil University of Medical Sciences, Ardabil, Iran

Corresponding Author: Karim Jafari, Department of Prosthodontics, Faculty of Dentistry, Ardabil University of Medical Sciences, Ardabil, Iran, Phone: +98 9123269848, e-mail: K.jafari@arums.ac.ir

How to cite this article: Farhang R, Hekmatfar S, Samadi V, et al. Comparison of Tooth Discoloration Induced by Calcium-enriched Mixture, Mineral Trioxide Aggregate, and Endocem. World J Dent 2020;11(5):392-395.

Source of support: The study was supported by Ardabil University of Medical Sciences.

Conflict of interest: None

low cytotoxicity, high biocompatibility, and good sealing ability. The CEM cement induces higher antibacterial effect, compared to MTA, due to entailing a higher level of alkaline. ${ }^{13,15}$ Results of previous investigations addressing the incidence of dental discoloration as a result of CEM cement have been controversial. Esmaeili et al. found that the CEM cement-induced discoloration was at the same level of that of the controls. ${ }^{16}$

Limited studies have evaluated tooth discoloration caused by CEM cement and Endocem in comparison with MTA. Therefore, the purpose of this study was to investigate the amount of crown

(c) The Author(s). 2020 Open Access This article is distributed under the terms of the Creative Commons Attribution 4.0 International License (https://creativecommons. org/licenses/by-nc/4.0/), which permits unrestricted use, distribution, and non-commercial reproduction in any medium, provided you give appropriate credit to the original author(s) and the source, provide a link to the Creative Commons license, and indicate if changes were made. The Creative Commons Public Domain Dedication waiver (http://creativecommons.org/publicdomain/zero/1.0/) applies to the data made available in this article, unless otherwise stated. 
discoloration as a result of applying CEM and mineral trioxide aggregate.

\section{Materials and Methods}

This experimental study was performed on 54 healthy central incisors extracted due to orthodontic treatment or periodontal disease. All specimens were screened to ensure the absence of restoration, cracks, caries, calcification, and discoloration. For the purpose of the study, the external surfaces of the teeth were cleaned by means of rubber cap and pumice, kept in $0.05 \%$ thymol solution for disinfection, and then maintained in a physiological saline solution to be applied later on.

Furthermore, the crown part of the tooth was cut with a high-speed diamond disk and abundant water $2 \mathrm{~mm}$ below the cementoenamel junction. The pulp remnants were removed by an excavator, and the pulp tissues were removed from the apical part of the cavity. The prepared cavity was washed with $2 \%$ sodium hypochlorite for 30 seconds and then dried with an absorbent point.

The samples were assigned into three groups of 18 members, namely group I (Endocem), group II (CEM cement; BioniqueDent, Tehran, Iran), and group III (MTA; Angelus MTA, Angelus, Londrina, Parana, Brazil) using simple random sampling technique. In each group, a $3 \mathrm{~mm}$-thick material was placed in the access cavity. Then, the samples were sealed by glass ionomer cement (Fuji II, GC, Japan). Tooth preparation and labeling was done by one person, and color change examination was done by another operator who did not know the labels of each sample.

To standardize for color assessment, a custom silicone matrix was prepared with impression material (speedex, colteenwhaledent, swiss) for each tooth.

The measurements were performed under constant laboratory illumination by the spectrophotometer (Vita EasyShade ${ }^{\circledast}$ compact, VITA Zahnfabrik, Germany) using in "tooth single" mode for all color evaluations.

The color measurements were repeated twice for each sample, and the same operator performed all color evaluations. Spectrophotometer measurements were obtained for each sample before material placement at the start as baseline (T1), 1 month (T2), and 3 months (T3) after application of endodontic materials.

Data were reported by using the $\operatorname{CIE} L^{*} a^{*} b^{*}$ system. The value of $L^{*}$ is the lightness. The values of $a^{*}$ and $b^{*}$ are the chromaticity coordinates in the red-green axis and the yellow-blue axis, respectively. The comparison of the measured $L^{*} a^{*} b^{*}$ values is expressed as $\Delta E$ calculated according following formula:

$$
\Delta E=\left[(\Delta L *)^{2}+(\Delta a *)^{2}+(\Delta b *)^{2}\right]^{1 / 2}
$$

Data analysis was performed using SPSS software (version 23) using one-way analysis of variance (ANOVA), repeated measures ANOVA, and post hoc tests. In addition, the normality of the data was evaluated using the Kolmogorov-Smirnov test.

\section{Result}

Table 1 presents the $\Delta E$ values obtained for the research groups at different periods of time. The results revealed a $\Delta E$ value of $>3.3$ in the three groups 1 month post-intervention. The KolmogorovSmirnov test showed that the data in all groups had normal distribution. Repeated-measures ANOVA showed the significant effect of time on color change ( $p$ value $=0.001$; Table 2 ). In addition,
Table 1: Mean and standard deviation of each material used in T1-30, T30-90, and T1-90 deltas

\begin{tabular}{lccr}
\hline Materials & Delta T1-T30 & Delta T30-T90 & Delta T1-T90 \\
\hline Endocem & $11.17 \pm 0.79$ & $14.040 \pm 0.1$ & $16.950 \pm 0.83$ \\
Angelus & $6.670 \pm 0.6$ & $11.420 \pm 0.88$ & $14.48 \pm 0.58$ \\
CEM cement & $6.86 \pm 0.63$ & $10.35 \pm 1.1$ & $14.360 \pm 0.99$ \\
\hline
\end{tabular}

T1, time to start MTA application; T30, one month after MTA application; T90, three months after MTA application

the results of one-way ANOVA revealed a significant difference between the groups a month after the application of materials ( $p$ value $=0.001$; Table 3 ). The highest color change was related to Endocem, and the least color change was observed in CEM cement.

\section{Discussion}

As the results of the present study indicated, all materials under investigation caused tooth discoloration, compared to basic color, during the first month. In the present study, extracted incisors were selected to be investigated because of their location in the esthetic zone. Tooth discoloration is a major esthetic concern for dental practitioners, especially when located on anterior teeth. Despite the limitation of extracted tooth model in the proper representation of the clinical and biological conditions of vital pulp, they are used to examine tooth discoloration resulting from endodontic materials in many studies. ${ }^{16,17}$

Determination of color can be accomplished via the application of visual methods or use of such instruments as spectrophotometer or colorimeter. The instrument utilized in the current study was the VITA Easy shade device with a reliable method of reading tooth color shade and high accuracy. ${ }^{18}$

The CEM cement contains metal oxides, such as calcium oxide, calcium phosphate, calcium carbonate, calcium silicate, calcium sulfate, calcium hydroxide, and calcium chloride. ${ }^{19}$ Chemical compositions of the CEM cement lack any iron (Fe) or bismuth oxide in contrast to the two types of MTA. ${ }^{20}$ The present study revealed no significant difference between the CEM cement and MTA samples in terms of tooth color change after 3 months. However, the samples subjected to CEM cement showed the lowest degree of discoloration. The difference in the discoloration of materials used in this study might be related to their chemical compositions.

Esmaeili et al. ${ }^{16}$ reported significant color changes even a week after the placement of CEM cement, MTA, and calcium hydroxide. In the mentioned study, CEM cement induced a lower level of discoloration when compared to MTA in all the time intervals tested. Araghi et al. ${ }^{21}$ reported that Biodentine caused tooth discoloration during 3 months. Furthermore, discoloration in MTA and CEM cement was similar but lower than that of Biodentin. Rouhani et al. ${ }^{17}$ investigated tooth color change after applying MTA and CEM cement and reported that MTA caused greater discoloration than CEM cement after 6 months. This controversy in the results presented in the literature may be due to the time of assessment (i.e., 6 months in their study vs 3 months in ours).

According to the results of several studies, after 6 months, the materials penetrate into the dentinal tubules, thereby resulting in discoloration. ${ }^{22,23}$ In addition, Parsons et al. ${ }^{24}$ found that discoloration was most evident at 9 and 12 months intervals, whereas the 1 and 3 months intervals exhibited minimal or no discoloration. The MTA is available in gray and white forms. White 
Tooth Discoloration Induced by CEM Cement, MTA and Endocem

Table 2: Results of one-way ANOVA test

\begin{tabular}{|c|c|c|c|c|c|c|}
\hline & & Sum of squares & $d f$ & Mean square & $F$ & Sig. \\
\hline \multirow[t]{3}{*}{ Delta 1 (T1-T30) } & Between groups & 233.514 & 2 & 116.757 & 13.852 & 0.037 \\
\hline & Within groups & 429.859 & 51 & 8.429 & & \\
\hline & Total & 663.373 & 53 & & & \\
\hline \multirow[t]{3}{*}{ Delta 2 (T30-T90) } & Between groups & 343.510 & 2 & 171.755 & 9.513 & 0.023 \\
\hline & Within groups & 920.824 & 51 & 18.055 & & \\
\hline & Total & 1264.334 & 53 & & & \\
\hline \multirow[t]{3}{*}{ Delta 3 (T1-T90) } & Between groups & 62.361 & 2 & 31.180 & 2.559 & 0.087 \\
\hline & Within groups & 621.452 & 51 & 12.185 & & \\
\hline & Total & 683.813 & 53 & & & \\
\hline
\end{tabular}

Table 3: Results of the post hoc comparison of groups

\begin{tabular}{|c|c|c|c|c|c|c|c|}
\hline \multicolumn{8}{|c|}{ Multiple comparisons } \\
\hline \multicolumn{8}{|c|}{ Tukey HSD } \\
\hline \multirow{2}{*}{$\begin{array}{l}\text { Dependent } \\
\text { variable }\end{array}$} & \multirow[b]{2}{*}{ (I) Materials } & \multirow[b]{2}{*}{ (J) Materials } & \multirow{2}{*}{$\begin{array}{l}\text { Mean difference } \\
(I-J)\end{array}$} & \multirow[b]{2}{*}{ Std. error } & \multirow[b]{2}{*}{ Sig. } & \multicolumn{2}{|c|}{ 95\% confidence interval } \\
\hline & & & & & & Lower bound & Upper bound \\
\hline \multirow{6}{*}{$\begin{array}{l}\text { Delta } 1 \text { (T1- } \\
\text { T30) }\end{array}$} & \multirow[t]{2}{*}{ Endocem } & Angelus & $4.502484^{*}$ & 0.967735 & 0.000 & 2.16639 & 6.83858 \\
\hline & & Cemcement & $4.314055^{*}$ & 0.967735 & 0.000 & 1.97796 & 6.65015 \\
\hline & \multirow[t]{2}{*}{ Angelus } & Endocem & $-4.502484^{*}$ & 0.967735 & 0.000 & -6.83858 & -2.16639 \\
\hline & & Cemcement & -0.188429 & 0.967735 & 0.979 & -2.52452 & 2.14766 \\
\hline & \multirow[t]{2}{*}{ Cemcement } & Endocem & $-4.314055^{*}$ & 0.967735 & 0.000 & -6.65015 & -1.97796 \\
\hline & & Angelus & 0.188429 & 0.967735 & 0.979 & -2.14766 & 2.52452 \\
\hline \multirow{6}{*}{$\begin{array}{l}\text { Delta } 2 \text { (T30- } \\
\text { T90) }\end{array}$} & \multirow[t]{2}{*}{ Endocem } & Angelus & $4.733182^{*}$ & 1.416387 & 0.004 & 1.31405 & 8.15231 \\
\hline & & Cemcement & $5.805134^{*}$ & 1.416387 & 0.000 & 2.38600 & 9.22426 \\
\hline & \multirow[t]{2}{*}{ Angelus } & Endocem & $-4.733182^{*}$ & 1.416387 & 0.004 & -8.15231 & -1.31405 \\
\hline & & Cemcement & 1.071952 & 1.416387 & 0.731 & -2.34718 & 4.49108 \\
\hline & \multirow[t]{2}{*}{ Cemcement } & Endocem & $-5.805134^{*}$ & 1.416387 & 0.000 & -9.22426 & -2.38600 \\
\hline & & Angelus & -1.071952 & 1.416387 & 0.731 & -4.49108 & 2.34718 \\
\hline \multirow{6}{*}{$\begin{array}{l}\text { Delta } 3 \text { (T1- } \\
\text { T90) }\end{array}$} & \multirow[t]{2}{*}{ Endocem } & Angelus & 0.866942 & 1.163583 & 0.738 & -1.94192 & 3.67581 \\
\hline & & Cemcement & 2.585921 & 1.163583 & 0.077 & -0.22294 & 5.39479 \\
\hline & \multirow[t]{2}{*}{ Angelus } & Endocem & -0.866942 & 1.163583 & 0.738 & -3.67581 & 1.94192 \\
\hline & & Cemcement & 1.718980 & 1.163583 & 0.310 & -1.08989 & 4.52785 \\
\hline & \multirow[t]{2}{*}{ Cemcement } & Endocem & -2.585921 & 1.163583 & 0.077 & -5.39479 & 0.22294 \\
\hline & & Angelus & -1.718980 & 1.163583 & 0.310 & -4.52785 & 1.08989 \\
\hline
\end{tabular}

*The mean difference is significant at 0.05 level.

MTA was introduced to reduce the concern regarding tooth discoloration. However, several reports indicated that even white MTA induces tooth discoloration. . $^{25} 26$

White MTA has lower concentrations of periclase (MgO), carborundum $\left(\mathrm{Al}_{2} \mathrm{O}_{3}\right)$, and FeO, in comparison with gray MTA; however, it still contains other metal oxides. ${ }^{9}$ The exact etiology and mechanism of discoloration are not clearly determined yet. However, there are a number of hypotheses regarding the cause of MTA-induced tooth discoloration. For instance, one hypothesis refers to the reaction of collagen present in organic dentin matrix with bismuth oxide. ${ }^{25}$

Shokouhinejad ${ }^{27}$ reported that sealing the pulp chamber walls with dentin bonding agent resulted in the reduction of MTA-induced tooth discoloration. It seems that the amino acids present in dentin collagen lead to the destabilization of bismuth oxide molecule in MTA. When the pulp chamber dentinal walls are sealed with dentin bonding agent leading, it can prevent from the migration of bismuth to the tooth structure, thereby resulting in less color change. ${ }^{25}$
In the current study, the discoloration induced by Endocem was significantly higher than that emanated from MTA and CEM cement in the first month. Endocem-treated samples presented similar discoloration regardless of the MTA and CEM cement after 3 months. Endocem is a newly developed pozzolan-based cement. Despite the similar chemical composition of Endocem and MTA, the former can be easily manipulated and has fast setting ability. These beneficial effects are the results of small pozzolanic particles. ${ }^{12}$ The pozzolanic reaction takes place between calcium hydroxide, as a cement hydration product, and pozzolan. ${ }^{28}$ Evaluation of the mineralization potential of Endocem by means of osteogenic differentiation markers revealed comparable results with those of ProRoot MTA. ${ }^{29}$

Jang et al. ${ }^{5}$ observed the evidence of color change over the first week of applying Endocem, which can be caused by the grayish color of the material. After 3 months, Endocem specimens presented little or no discoloration. In our study, there was no significant difference in the discoloration of all materials after 3 
months. Yun et al. investigated tooth discoloration emerged as a results of contacting with various calcium-silicate-based pulp capping materials. They found that Pro root MTA and Endocem caused more discoloration than control group. Presence of bismuth oxide in these materials might be a possible explanation for the results. ${ }^{30}$ It is required to perform clinical studies using cements in contact with the vital tissues to verify the results of in vitro studies and effect of endodontic materials on the color of the teeth.

\section{Conclusion}

The results of the current study showed a significant difference between tooth discoloration a month after applying CEM cement, MTA, and Endocem.

\section{References}

1. Ahmed $H$, Abbott P. Discolouration potential of endodontic procedures and materials: a review. Int Endod J 2012;45(10):883-897. DOI: 10.1111/j.1365-2591.2012.02071.x.

2. Tawil PZ, Trope M, Curran AE, et al. Periapical microsurgery: an in vivo evaluation of endodontic root-end filling materials. J Endod 2009;35(3):357-362. DOI: 10.1016/j.joen.2008.12.001.

3. Vajrabhaya LO, Korsuwannawong S, Jantarat J, et al. Biocompatibility of furcal perforation repair material using cell culture technique: Ketac molar vs ProRoot MTA. Oral Surg Oral Med Oral Pathol Oral Radiol Endod 2006;102(6):e48-e50. DOI: 10.1016/j.tripleo.2006.05.015.

4. Guo YJ, Du TF, Li HB, et al. Physical properties and hydration behavior of a fast-setting bioceramic endodontic material. BMC Oral Health 2016;16(1):23. DOI: 10.1186/s12903-016-0184-1.

5. Jang JH, Kang M, Ahn S, et al. Tooth discoloration after the use of new pozzolan cement (Endocem) and mineral trioxide aggregate and the effects of internal bleaching. J Endod 2013;39(12):1598-1602. DOI: 10.1016/j.joen.2013.08.035.

6. Parirokh $M$, Torabinejad M, Dummer P. Mineral trioxide aggregate and other bioactive endodontic cements: an updated overview-part I: vital pulp therapy. Int Endod J 2018;51(2):177-205. DOI: 10.1111/ iej.12841.

7. Bortoluzzi EA, Araújo GS, Tanomaru JMG, et al. Marginal gingiva discoloration by gray MTA: a case report. J Endod 2007;33(3):325-327. DOI: 10.1016/j.joen.2006.09.012.

8. Karabucak B, Li D, Lim J, et al. Vital pulp therapy with mineral trioxide aggregate. Dental Traumatol 2005;21(4):240-243. DOI: 10.1111/j.16009657.2005.00306.x

9. Asgary S, Parirokh M, Eghbal MJ, et al. Chemical differences between white and gray mineral trioxide aggregate. J Endod 2005;31(2):101103. DOI: 10.1097/01.DON.0000133156.85164.B2.

10. Belobrov I, Parashos P. Treatment of tooth discoloration after the use of white mineral trioxide aggregate. J Endod 2011;37(7):1017-1020. DOI: 10.1016/j.joen.2011.04.003.

11. Kang SH, Shin YS, Lee HS, et al. Color changes of teeth after treatment with various mineral trioxide aggregate-based materials: an ex vivo study. J Endod 2015;41(5):737-741. DOI: 10.1016/j.joen.2015.01.019.

12. Kim RJY, Kim MO, Lee KS, et al. An in vitro Evaluation of the antibacterial properties of three mineral trioxide aggregate (MTA) against five oral bacteria. Arch Oral Biol 2015;60(10):1497-1502. DOI: 10.1016/j.archoralbio.2015.07.014.

13. Esnaashari E, Pezeshkfar A, Fazlyab M. Nonsurgical management of an extensive perforative internal root resorption with calcium-enriched mixture cement. Iran Endod J 2015;10(1):75.
14. Madani ZS, Haddadi A, Mesgarani A, et al. Histopathologic responses of the dental pulp to calcium-enriched mixture (CEM) and mineral trioxide aggregate (MTA) in diabetic and non-diabetic rats. Int J Mol Cell Med 2014;3(4):263.

15. Asgary $\mathrm{S}$, Shahabi $\mathrm{S}$, Jafarzadeh $\mathrm{T}$, et al. The properties of a new endodontic material. J Endod 2008;34(8):990-993. DOI: 10.1016/j. joen.2008.05.006.

16. Esmaeili B, Alaghehmand $\mathrm{H}$, Kordafshari $\mathrm{T}$, et al. Coronal discoloration induced by calcium-enriched mixture, mineral trioxide aggregate and calcium hydroxide: a spectrophotometric analysis. Iran Endod J 2016;11(1):23

17. Rouhani A, Akbari M, Farhadi-faz A. Comparison of tooth discoloration induced by calcium-enriched mixture and mineral trioxide aggregate. Iran Endod J 2016;11(3):175.

18. Kim-Pusateri S, Brewer JD, Davis EL, et al. Reliability and accuracy of four dental shade-matching devices. J Prosthe Dent 2009;101(3):193199. DOI: 10.1016/S0022-3913(09)60028-7.

19. Asgary S, Ahmadyar M. Vital pulp therapy using calcium-enriched mixture: an evidence-based review. J Conserv Dent 2013;16(2):92. DOI: 10.4103/0972-0707.108173.

20. Mozayeni MA, Milani AS, Marvasti LA, et al. Cytotoxicity of calcium enriched mixture cement compared with mineral trioxide aggregate and intermediate restorative material. Aust Endod J 2012;38(2):70-75. DOI: 10.1111/j.1747-4477.2010.00269.x.

21. Araghi S, Khavid A, Godiny M, et al. In vitro evaluation of coronal discoloration following the application of calcium-enriched mixture cement, Biodentine, and mineral trioxide aggregate in endodontically treated teeth. Dent Res J (Isfahan) 2019;16(1):53. DOI: 10.4103/1735-3327.249550.

22. Arman $M$, Khalilak $Z$, Rajabi $M$, et al. In vitro spectrophotometry of tooth discoloration induced by tooth-colored mineral trioxide aggregate and calcium-enriched mixture cement. Iran Endod J 2015;10(4):226.

23. Rao YM, Srilakshmi V, Vinayagam KK, et al. An Evaluation of the color stability of tooth-colored restorative materials after bleaching using CIELAB color technique. Indian J Dent Res 2009;20(1):60. DOI: 10.4103/0970-9290.49071.

24. Parsons JR, Walton RE, Ricks-Williamson L. In vitro longitudinal assessment of coronal discoloration from endodontic sealers. J Endod 2001;27(11):699-702. DOI: 10.1097/00004770-200111000-00012.

25. Marciano MA, Costa RM, Camilleri J, et al. Assessment of color stability of white mineral trioxide aggregate angelus and bismuth oxide in contact with tooth structure. J Endod 2014;40(8):1235-1240. DOI: 10.1016/j.joen.2014.01.044.

26. Ioannidis K, Mistakidis I, Beltes $\mathrm{P}$, et al. Spectrophotometric analysis of coronal discolouration induced by grey and white MTA. Int Endod J 2013;46(2):137-144. DOI: 10.1111/j.1365-2591.2012.02098.x.

27. Shokouhinejad N, Razmi H, Farbod M, et al. Coronal tooth discoloration induced by regenerative endodontic treatment using different scaffolds and intracanal coronal barriers: a 6 month ex vivo study. Restor Dent Endod 2019;44(3):e25. DOI: 10.5395/rde.2019.44.e25.

28. Askarinejad A, Pourkhorshidi AR, Parhizkar T. Evaluation the pozzolanic reactivity of sonochemically fabricated nano natural pozzolan. Ultrason Sonochem 2012;19(1):119-124. DOI: 10.1016/j. ultsonch.2011.05.005.

29. Choi Y, Park SJ, Lee SH, et al. Biological effects and washout resistance of a newly developed fast-setting pozzolan cement. J Endod 2013;39(4):467-472. DOI: 10.1016/j.joen.2012.11.023.

30. Yun DA, Park SJ, Lee SR, et al. Tooth discoloration induced by calciumsilicate-based pulp-capping materials. Eur J Dent 2015;9(2):165. DOI: 10.4103/1305-7456.156789. 